\title{
Salvage of an exposed cranial prosthetic implant using a transposition flap with an indwelling antibiotic irrigation system
}

\author{
Sung Oh Hwang, \\ Lan Sook Chang \\ Department of Plastic and \\ Reconstructive Surgery, Inje University \\ Ilsan Paik Hospital, Inje University \\ College of Medicine, Goyang, Korea
}

\begin{abstract}
Cranial implant removal is recommended if implants become exposed owing to scalp necrosis after cranioplasty. However, it carries the risk of extensive bleeding, and the resultant cranial defects can cause both aesthetic and functional problems. We present a case of a scalp defect exposing a cranial prosthetic implant that was reconstructed with a local flap and salvaged using an indwelling antibiotic irrigation system. A 73-year-old man presented with scalp necrosis after undergoing cranioplasty due to intracranial hemorrhage. The cranial implant was exposed through the scalp defect. Methicillin-resistant Staphylococcus aureus was detected in the culture from the open wound. After debridement of the necrotic tissue and burring of the superficial layer of the implant, a transposition flap was used to cover the defect and an indwelling antibiotic irrigation system was installed. Continuous irrigation with vancomycin was conducted for 5 days, and intravenous vancomycin was continued for 4 weeks. The flap was in good condition at 4 months postoperatively, with no infection. The convex contour of the scalp was well maintained. The patient's neurological status was stable. Exposed cranial implants can be salvaged with continuous antibiotic irrigation as an alternative to implant removal; thus, the risk of bleeding and possible disfigurement may be avoided.
\end{abstract}

Keywords: Indwelling catheter / Prosthetic and implants / Salvage treatment / Therapeutic irrigations

\section{INTRODUCTION}

Scalp necrosis, which is one of the most serious complications of cranioplasty, can be caused by poor blood circulation $[1,2]$. If scalp necrosis progresses to the full thickness of the tissue and the underlying bone flap or prosthetic implant is exposed, removal of the implant material (autologous or prosthetic) is generally recommended [3]. However, various problems can result

\footnotetext{
Correspondence: Lan Sook Chang

Department of Plastic and Reconstructive Surgery, Inje University Ilsan Paik Hospital, Inje University College of Medicine, 170 Juhwa-ro, Ilsanseo-gu, Goyang 10380, Korea

E-mail: aseret@naver.com
}

Received November 4, 2019 / Revised November 27, 2019 / Accepted December 4, 2019 from the cranial defects that occur after removal of the implant material. In addition to the cosmetic problems caused by cranial disfigurement, functional problems associated with cerebrospinal fluid (CSF) hemodynamics, brain protection, and sinking skin flap syndrome can occur [4]. The removal of the implant material itself can lead to extensive bleeding because of dura adhesions, which is a particularly concerning risk for patients in poor general condition. If the signs of infection are not clear or the infection has a subtle course, scalp reconstruction while preserving the previous implant is an alternative method worth considering.

Here, we present a case of a large scalp defect exposing a prosthetic cranial implant that was successfully salvaged through 
transposition flap reconstruction while controlling infection with an indwelling antibiotic irrigation system.

\section{CASE REPORT}

A 73-year-old man underwent cranioplasty with a polyethylene implant (Medpor; Stryker Instruments, Portage, MI, USA) after craniectomy due to intracranial hemorrhage 3 months before presenting at our hospital. Poor blood circulation due to extensive tension led to full-thickness necrosis of the scalp flap. The cranial implant was exposed through a temporal scalp defect measuring $10 \times 8 \mathrm{~cm}$ (Fig. 1).

Methicillin-resistant Staphylococcus aureus was cultured from the open wound, and the preoperative C-reactive protein level was $1.8 \mathrm{mg} / \mathrm{dL}$. Brain magnetic resonance imaging revealed dural enhancement, indicating the need for the differential diagnosis of a possible infectious condition based on the postoperative changes. However, the patient's clinical symptoms were mild, with minimal discharge and no fever. After consulting a neurosurgeon, a decision was made to salvage the implant considering the patient's advanced age and poor general condition. The patient was in a bedridden state, was unable to communicate, and presented with underlying atrial fibrillation.

Surgery was performed under general anesthesia. The scalp surrounding the defect was widely excised. Burring of the superficial surface of the Medpor implant was performed, and the exposed plates and screws were changed, followed by copious

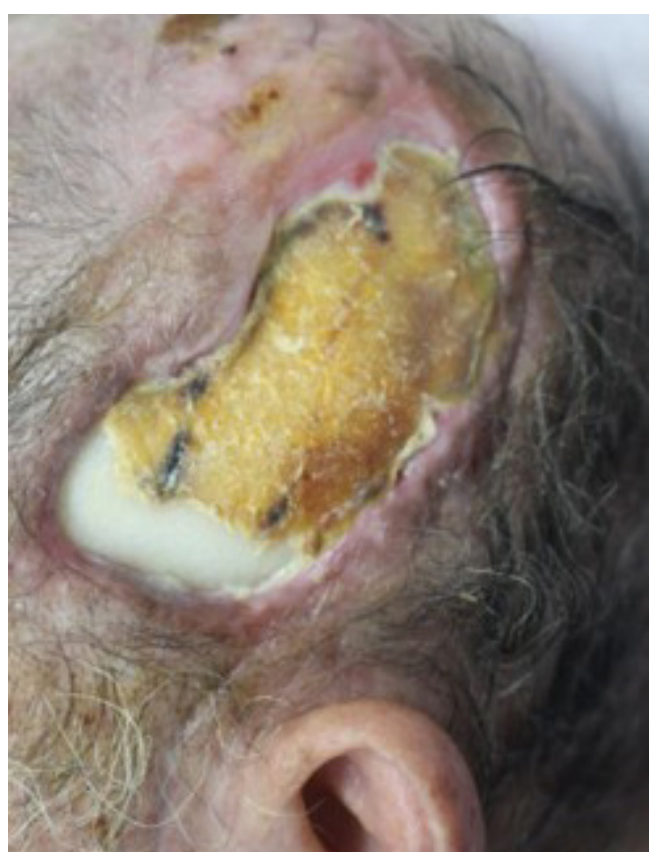

Fig. 1. A previously inserted cranial implant was exposed through a temporal scalp defect measuring $10 \times 8 \mathrm{~cm}$. irrigation. A large transposition flap was elevated from the occipital area in the subgaleal plane and inset into the defect. The donor site was covered with split-thickness skin grafts from the anterolateral thigh (Fig. 2). An indwelling antibiotic irrigation system was installed using an intravenous line set. The line was connected to the antibiotic solution and was inserted through

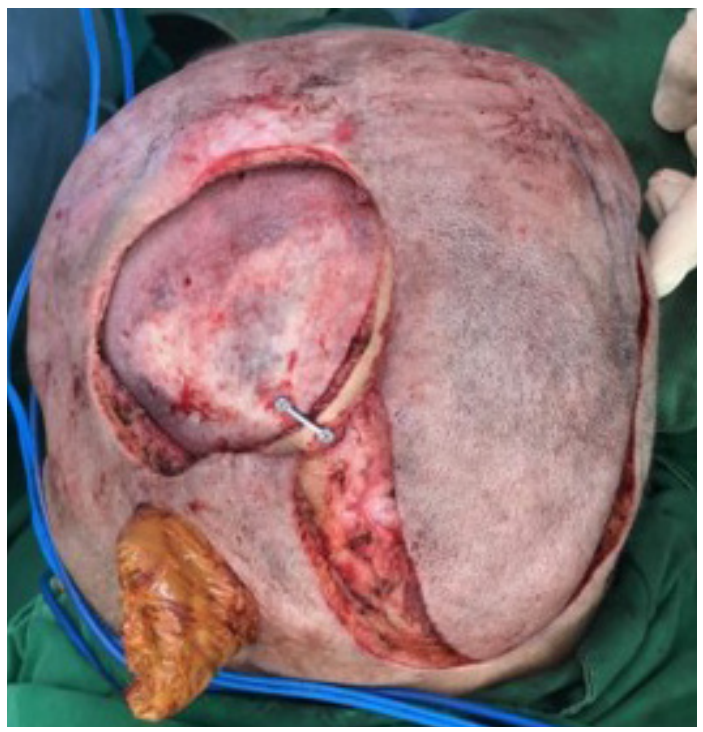

Fig. 2. The scalp surrounding the defect was widely excised. Burring of the superficial surface of the Medpor implant was performed and the exposed plates and screws were changed, followed by copious irrigation. A large transposition flap was elevated from the occipital area in the subgaleal plane and inset into the defect.

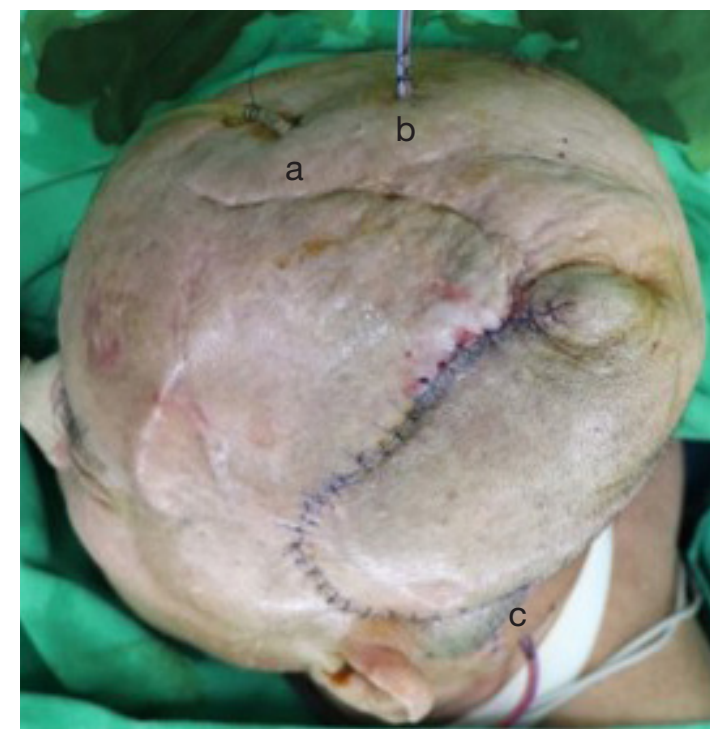

Fig. 3. An indwelling antibiotic irrigation system was installed using an intravenous line set. Line a, which was connected with the antibiotic solution, was inserted through frontal scalp and served as an inlet, and two Hemovac drains (b, c) were inserted and served as outlets. 


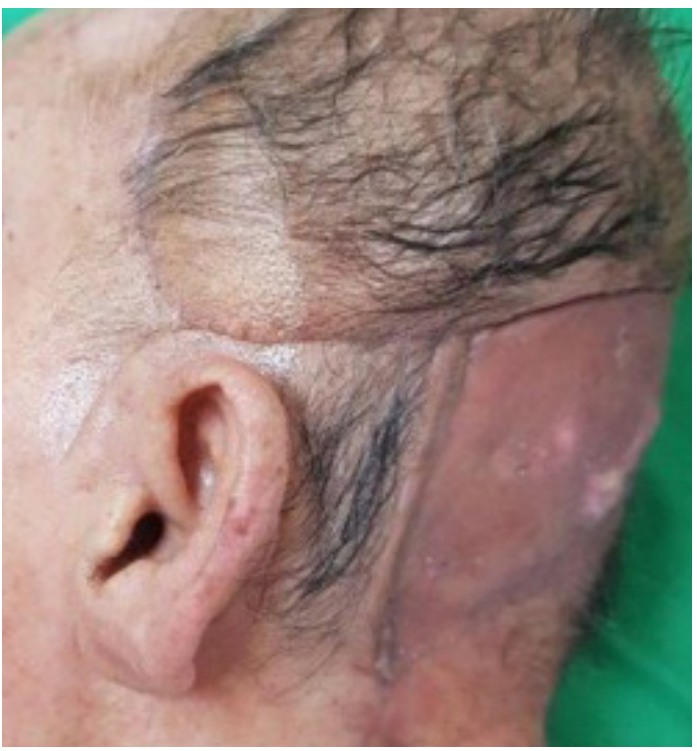

Fig. 4. The flap was in good condition without evidence of infection at a 4 -month postoperative follow-up visit.

the frontal scalp, which served as an inlet, and two drains (Baro-Vac; Sewoon Medical, Cheonan, Korea) were inserted in the dependent position as outlets (Fig. 3). After confirming the patency of the irrigation system, a vancomycin solution at a concentration of $1,000 \mathrm{mg} / \mathrm{L}$ was continuously infused through the system at an infusion rate of $20 \mathrm{~mL} / \mathrm{hr}$ for 5 days. Intravenous vancomycin was also administered for 4 weeks postoperatively. The irrigation system was removed at 1 week postoperatively, and the Hemovac drain was maintained until the drain volume was $<10 \mathrm{~mL} /$ day.

The flap was in good condition without evidence of infection at a 4-month postoperative follow-up visit (Fig. 4). However, the patient died from cardiac arrest, presumably due to a cardiac problem that arose in the weeks after wound healing.

\section{DISCUSSION}

Infection after cranioplasty is uncommon; however, delayed infection is one of the most critical complications of cranioplasty [5-7]. In the worst-case scenario, central nervous system infections can occur. In such cases, the standard treatment is removal of the implant material [3].

However, after removing the implant material, cranial disfigurement can occur due to severe craniofacial asymmetry, which is not aesthetically pleasing, and the resultant altered physical appearance can be traumatic for the patient. Moreover, cranial disfigurement can impede the patient's return to daily life and occupation [8]. In addition, the presence of a cranial defect may make it impossible to protect the brain, and the possibility of brain damage is increased. Hence, protective equipment such as a helmet is required $[9,10]$. Without the underlying implant material, the scalp would sink under atmospheric pressure, thereby applying pressure to the cerebral cortex. This affects cerebral perfusion and CSF flow, leading to sinking skin flap syndrome and, occasionally, paradoxical herniation [4].

Many attempts have been made to retain the bone flap material for several reasons. Delgado-Lopez et al. [11] and Auguste and McDermott [12] succeeded in preserving a previous bone flap using an antibiotic irrigation system. This effective indwelling antibiotic irrigation system has also been shown to be useful for salvaging infected nasal cartilage, in addition to cranial bone flaps [13]. Our case differs from previous reports in which the prosthetic cranial implant was successfully salvaged with an antibiotic irrigation system along with scalp defect reconstruction. If the scalp wound underlying the implant material is found to be inflamed or severely infected, the implant should be removed. In the present case, however, preservation of the implant could be attempted owing to the subtle course of the infection. Furthermore, the patient's general condition and the risk posed by implant removal surgery were considered. An abundant blood supply through flap coverage may also improve the ability to control infection.

In conclusion, salvage of an exposed prosthetic cranial implant using indwelling antibiotic irrigation provides a relatively simple and cost-effective option. Owing to its distinct benefits and acceptable outcomes, we recommend that preservation of prosthetic cranial implants using an indwelling antibiotic irrigation system should be considered first in certain patients before resorting to implant removal.

\section{NOTES}

\section{Conflict of interest}

No potential conflict of interest relevant to this article was reported.

\section{Ethical approval}

The study was performed in accordance with the principles of the Declaration of Helsinki. Written informed consent was obtained.

\section{Patient consent}

The patient provided written informed consent for the publication and the use of his images.

\section{ORCID}

Sung Oh Hwang https://orcid.org/0000-0001-5537-9124 
Lan Sook Chang https://orcid.org/0000-0003-4725-772X

\section{REFERENCES}

1. Di Rienzo A, Pangrazi PP, Riccio M, Colasanti R, Ghetti I, Iacoangeli M. Skin flap complications after decompressive craniectomy and cranioplasty: proposal of classification and treatment options. Surg Neurol Int 2016;7:S737-45.

2. Yang HS, Hyun D, Oh CH, Shim YS, Park H, Kim E. A faster and wider skin incision technique for decompressive craniectomy: $\mathrm{n}$-shaped incision for decompressive craniectomy. Korean J Neurotrauma 2016;12:72-6.

3. Hlavin M, Ratcheson R. Intracranial epidural abscess. In: Kaye $\mathrm{AH}$, Black PM, editors. Operative neurosurgery. London: Churchill Livingstone; 2000. p. 1679-85.

4. Kurland DB, Khaladj-Ghom A, Stokum JA, Carusillo B, Karimy JK, Gerzanich V, et al. Complications associated with decompressive craniectomy: a systematic review. Neurocrit Care 2015;23:292-304.

5. Edwards MS, Ousterhout DK. Autogeneic skull bone grafts to reconstruct large or complex skull defects in children and adolescents. Neurosurgery 1987;20:273-80.

6. Prolo DJ, Oklund SA. Composite autogeneic human cranioplasty: frozen skull supplemented with fresh iliac corticocan- cellous bone. Neurosurgery 1984;15:846-51.

7. Rish BL, Dillon JD, Meirowsky AM, Caveness WF, Mohr JP, Kistler JP, et al. Cranioplasty: a review of 1030 cases of penetrating head injury. Neurosurgery 1979;4:381-5.

8. Oikawa S, Mizuno M, Muraoka S, Kobayashi S. Retrograde dissection of the temporalis muscle preventing muscle atrophy for pterional craniotomy. Technical note. J Neurosurg 1996;84: 297-9.

9. Honeybul S. Decompressive craniectomy: a new complication. J Clin Neurosci 2009;16:727-9.

10. Wind JJ, Ohaegbulam C, Iwamoto FM, Black PM, Park JK. Immediate titanium mesh cranioplasty for treatment of postcraniotomy infections. World Neurosurg 2013;79:207.

11. Delgado-Lopez PD, Martin-Velasco V, Castilla-Diez JM, Galacho-Harriero AM, Rodriguez-Salazar A. Preservation of bone flap after craniotomy infection. Neurocirugia (Astur) 2009;20: 124-31.

12. Auguste KI, McDermott MW. Salvage of infected craniotomy bone flaps with the wash-in, wash-out indwelling antibiotic irrigation system: technical note and case series of 12 patients. J Neurosurg 2006;105:640-4.

13. Walton RL, Wu LC, Beahm EK. Salvage of infected cartilage grafts for nasal reconstruction with a through-and-through irrigation system. Ann Plast Surg 2005;54:445-9. 